\title{
Effects of Amino Acids on Olfactory-Related Receptors Regulating Appetite in Silver Pomfret
}

\author{
Youyi Zhang \\ Ningbo University \\ Jiabao Hu \\ Qijun Le \\ Ningbo University \\ Yang Yang \\ Ningbo University \\ Siwen Kuang \\ Ningbo University \\ Man Zhang \\ Ningbo University \\ Weiwei Gu \\ Ningbo University \\ Yibo Sun \\ Ningbo University \\ Kimran Jean Jacques \\ Ningbo University \\ Yaya Li \\ Ningbo University \\ Jiachu Sun \\ Ningbo University \\ Yajun Wang ( $\nabla$ wangyajun@nbu.edu.cn ) \\ Ningbo University \\ Chunjing Zheng \\ Ningbo University \\ Shanliang Xu \\ Ningbo University \\ Xiaojun Yan \\ Ningbo University
}

Ningbo University https://orcid.org/0000-0003-0970-1332 
Keywords: Amino acid, Olfactory receptor, Appetite, Phagostimulants, Transcriptome, Pampus argenteus Posted Date: October 7th, 2020

DOI: https://doi.org/10.21203/rs.3.rs-78165/v1

License: (1) (1) This work is licensed under a Creative Commons Attribution 4.0 International License. Read Full License 


\section{Abstract}

Background: Amino acids are common components of the natural prey of fishes, and even single amino acid can bind olfactory receptors of fishes and induce appetite, which is regulated by hormones. Silver pomfret (Pampus argenteus) is one of the most preferred commercial marine fish species in many Asian countries and performs a significant appetite for jellyfish. Aspartic acid, glycine, and cysteine are high content amino acid in jellyfish, so we investigated the effect of three amino acids (aspartic acid, glycine, and cysteine) on olfactory-related receptors regulating appetite in silver pomfret (Pampus argenteus).

Results: The fish showed significant attractant responses to these amino acids in water, which were also observed to improve the ingestion rates of the fish. Next, we conducted transcriptomes of the olfactory epithelium (OE) and identified 34 olfactory-related receptor genes were including olfactory receptors, trace amine-associated receptors and vomeronasal receptors genes, and we examined these genes in the OE and appetite-related genes (GHRL and $\angle E P$ in gut/stomach and $N P Y, A g R P$, and POMC in brain) by RTqPCR. The olfactory-related receptor genes were significantly expressed in amino acid groups, and the appetite-related genes were most significantly expressed in aspartic acid group.

Conclusions: Thus, olfactory-related receptors induced by amino acids might regulate appetite in silver pomfret through the OE-brain-gut/stomach axis. Using these data, we identified some effective amino acid phagostimulants which could be supplied in silver pomfret diet, and the results improved our understanding of the mechanism of olfactory-related receptors regulating appetite in fish.

\section{Background}

Amino acids are common components of the natural prey of fishes, and even in the presence of single amino acid, olfactory receptors of fishes can bind it and trigger feeding behaviors (Hara, 2010, Franklyn et al., 2017). Therefore, amino acids are considered good phagostimulants when mixed with feed (Hu et al., 2017, Bell et al., 1996), and these phagostimulants can significantly affect the appetite of fish through their olfactory neural circuitry (Tandler et al., 1982, Koide et al., 2009). Odorant molecules released into the environment bind to olfactory-related receptors (Kaupp, 2010). Until now, three types of olfactoryrelated receptors have been identified in the olfactory epithelium of fishes, including olfactory receptors (ORs) (Johnstone et al., 2012), vomeronasal receptors (VRs) (Niimura, 2009), and trace amine-associated receptors (TAARs) (Kolmakov et al., 2008). The first OR gene was identified in Rattus norvegicus. It encoded a G-protein-coupled receptor expressed in ciliated sensory neurons (Buck and Axel, 1991). Subsequently, V1Rs and V2Rs were also identified, and they are considered to be the main receptors for pheromones (Buck and Axel, 1991). In fishes, because of the lack of a vomeronasal system (Pfister and Rodriguez, 2005), VRs are located in microvillous sensory neurons in the olfactory epithelium (Cao et al., 1998, Asanomiyoahi et al., 2000). The TAARs were first identified in mouse olfactory epithelium, and they are required to recognize amines found in urine (Liberles and Buck, 2006); these receptors were also identified in zebrafish, Danio rerio, in 2005 (Gloriam et al., 2005). 
Phagostimulants activate olfactory-related receptors to induce appetite, which is regulated by hormones (Mackenzie et al., 1998, Hu et al., 2019), and these appetite-regulating hormones are mainly secreted by the brain (hypothalamus and central nervous system) and gastrointestinal tissues (peripheral organs) (Bertucci et al., 2019). Of the appetite-regulating hormones secreted by the central nervous system of fish, neuropeptide Y (NPY) - which regulates food intake (Campos et al., 2010, Helene, 2016)-and agoutirelated protein (AgRP) - which plays an essential role in feeding stimulation-are mainly expressed in the brain and pituitary (Miguel and Ector, 2003, Volkoff, 2016), whereas proopiomelanocortin (POMC) is an anorectic factor that suppresses appetite in fish (Volkoff, 2016). Of the appetite-regulating hormones expressed in the gastrointestinal tissues, ghrelin (GHRL) - which is mainly released in the stomach and gut-plays an orexigenic role in improving feed intake (Cummings et al., 2002), whereas leptin (LEP) is an important anorectic factor that regulates the expression of other anorexigenic neuropeptides such as POMC (Gong et al., 2015). However, there is limited research on the relationship between olfactory and appetite in fish.

Silver pomfret (Pampus argenteus) is one of the most preferred commercial marine fish species in many Asian countries. Recently, the silver pomfret catch has greatly reduced because of overfishing, and the culture and breeding of this fish are faced with many challenges. In 2018, we succeeded in breeding approximately a million young silver pomfret; however, we still need to solve the problem of feeding efficiency. In the culturing process, we found that the fish showed a significant appetite for jellyfish including Aurelia aurita and Rhopilema esculentum, which had been reported in previous studies (Si et al., 2015, Liu et al., 2015). In the present study, we investigated whether the amino acids in jellyfish affected the olfactory-related receptors regulating appetite in silver pomfret by using four different concentrations of three amino acids and betaine and testing their attractant effects in fish diets. After identifying the optimal concentration, we examined how the exposure to amino acids affected the expression of olfactory-related genes by transcriptomic analysis and the expression of appetite-regulating genes by RTqPCR. Using these data, we identified some amino acid phagostimulants which could be supplied and partly replace the jellyfish in silver pomfret diet; our results improve the understanding of the mechanism of olfactory-related receptors regulating appetite in fish.

\section{Methods}

\subsection{Determination of optimal amino acid concentration and samples}

The juvenile silver pomfrets (Pampus argenteus) (mean weight: $12.99 \pm 1.10 \mathrm{~g}$; mean total length: $9.05 \pm$ $1.57 \mathrm{~cm}$ ) were reared at Xiangshan Bay, Zhejiang, China. All fish experiments were conducted in accordance with the recommendations of the National Institutes of Health Guide for the Care and Use of Laboratory Animals. The Animal Care and Use Committee of Ningbo University approved the protocols. The fish were fed with a commercial feed (larve love 6\#, Hayashikane Sangyo Co., Ltd., Yamaguchi-ken, Japan) mixed with fresh water (1:1 by weight). The total amount of feed provided was $2 \%$ of fish body 
weight 3 times per day (every $4 \mathrm{~h}$ ), and the fish were starved for $12 \mathrm{~h}$ before the experiments. The fish were randomly divided into different groups for experiments.

In accordance with the amino acid proportion of Rhopilema esculentum (Yu et al., 2014, Xiguang, 2004), we selected high-content amino acids in jellyfish and used $0.1 \mathrm{~mol} \mathrm{L-1}$ of these amino acids diluted in distilled water to stimulate the fish in pre-treatments; the amino acids were added to the culturing water slowly using an air stone tube with a $50 \mathrm{~mL}$ syringe (the air stone was submerged on the swimming route of the fish). It was observed that the fish were evidently attracted to L-Aspartic acid (Asp), L-Glycine (Gly) and L-Cysteine (Cys). Additionally, betaine (Bet; trimethylglycine)-known to be an effective amino acid phagostimulant in aquaculture (Hu et al., 2019) - was used as positive control, and fresh water was used as negative control (Control). The amino acid and betaine were purchased from Aladdin (Shanghai, China; purity $\geq 98 \%$ ). We found that the fish showed obviously attractive behaviors for $5 \mathrm{~min}$ in pretreatments, so the amino acid solutions were added to the culturing water slowly for $5 \mathrm{~min}$ in each pond (10 $\mathrm{mL}$ amino acids water was steadily added every $1 \mathrm{~min}$ ) in each group at the same time of the day.

To determine the optimal concentration of the three amino acids as feed attractant, four concentrations obtained by diluting the amino acids in fresh water $(0.06 \mathrm{~mol} \mathrm{L-1}$ [C1], $0.08 \mathrm{~mol} \mathrm{L-1}$ [C2], $0.1 \mathrm{~mol} \mathrm{L-1}$ [C3], and 0.12 mol L-1 [C4]) were used for the following test groups (Asp group, Gly group, and Cys group), and four concentrations of Bet were obtained by diluting it in fresh water $(0.06 \mathrm{~mol} / \mathrm{L}$ [C1], 1.1\% [C2], 1.4\% [C3], and $1.7 \%$ [C4]) for positive groups, and fresh water was used as negative control group (Control). The concentration levels referred to our previous studies (Franklyn et al., 2017, Hu et al., 2019).100 silver pomfret were cultured in a pond for 1 independent biological replicates (volume: 3000 L; depth: 1 m; $25 \pm$ $1{ }^{\circ} \mathrm{C} ; \mathrm{pH}, 8.21 \pm 0.3$; dissolved oxygen, $7.35 \pm 0.05 \mathrm{mg} \mathrm{L}-1$; salinity, $20.3 \pm 0.8 \%$; same 3 independent biological replicates for each group). The fish were stimulated 3 times per day (every 4 h; total 3 days), and the culture water were changed totally after every test.

The behavioral response of the fish was classified as two types: stopping swimming and biting the air stone (which was considered to be a positive feeding response) and swimming in close proximity of the air stone without biting (which was considered to be a positive movement response). The behaviors were recorded with a camera, and the number of each type of response was noted and then analyzed statistically using one-way analysis of variance (ANOVA) and Tukey 's multiple comparison test (SPSS, version 16.0). Based on the analysis of the number of fish per min (totally $5 \mathrm{~min}$ ) for the two behavioral responses, we selected the optimal concentration of amino acids and used the fish showing a positivefeeding response with this concentration for RNA sequencing.

The optimal concentration that produced the highest number of behavioral responses was used for subsequent stimulation experiments, which were performed as described as above. We stimulated the new no-test fish 3 times per day (every 4 h; total 3 days). In last time, we sunk a net table below the air stone in the bottom of pond, and we pulled up the net table fast and captured the fish which exhibited significantly positive feeding responses. Then, the fish was anaesthetized by MS-222 (Canton, Shanghai, China), and the olfactory epithelium (OE), brain (B), and gut/stomach (GS) tissues from 15 randomly 
selected fish (per replicate) were isolated, and each type of tissue was pooled into three $1.5 \mathrm{~mL}$ RNasefree tubes ( 3 independent biological replicates for each group) and stored in liquid nitrogen. The OE tissues were used for RNA-seq and real-time quantitative PCR (RT-qPCR), and the whole brain (B) and gut/stomach (GS) tissues were used for RT-qPCR.

\subsection{Verification of the effect of amino acids in feed}

To examine the effect of the optimal concentration of the three amino acids and Bet in the feed, we added them to commercial diet (larve love 6\#, Hayashikane Sangyo Co., Ltd, Japan) and fed to five groups of silver pomfret to determine whether it improved feeding performance. The nutritional composition of the commercial feed was shown in the table S1. The commercial feed was mixed with fresh water (1:1 by weight; diluted optimal concentration of amino acids and Bet) for test groups. For comparison, the control groups were fed feed mixed with fresh water (1:1 by weight; no amino acid supplementation). The feed was placed at the center of a feeding net table which was hung in water.

We cultured the new no-test fish in $3000 \mathrm{~L}$ ponds and starved the fish for $24 \mathrm{~h}$ before the test $(100$ individuals per pond; 3 independent biological replicates for each group). The fish were fed three times per day (every $4 \mathrm{~h}$; total 3 days), and the culture water were changed totally after every test. The total amount of feed provided was $2 \%$ of fish body weight each time. The time taken for the fish to consume the feed was recorded at every feeding and analyzed using one-way ANOVA and tukey's multiple comparison test (SPSS, version 16.0).

\subsection{RNA quantification and qualification}

Total RNA of tissues was extracted using the TRIzol reagent (Invitrogen, CA, USA). RNA degradation and contamination were monitored on $1 \%$ agarose gels. RNA purity was checked using the NanoPhotometer ${ }^{\circledR}$ spectrophotometer (IMPLEN, CA, USA). RNA concentration was measured using the Qubit® RNA Assay Kit and Qubit ${ }^{\circledR}$ 2.0 Fluorometer (Life Technologies, CA, USA). RNA integrity was assessed using the RNA Nano 6000 Assay Kit and Agilent Bioanalyzer 2100 system (Agilent Technologies, CA, USA).

\subsection{Library preparation for transcriptome sequencing}

A total of $1.5 \mu \mathrm{g}$ RNA per sample was used as the input material. Sequencing libraries were prepared using the NEBNext ${ }^{\circledR}$ Ultra ${ }^{\mathrm{T}}$ RNA Library Prep Kit for Illumina ${ }^{\circledR}$ (NEB, USA) according to the manufacturer's instructions, and index codes were added to attribute sequences to each sample. In brief, mRNA was purified from the total RNA by using poly-T oligo-attached magnetic beads. Fragmentation was performed using divalent cations under high temperature in NEBNext First Strand Synthesis Reaction Buffer (5x). First-strand cDNA was synthesized using random hexamer primers and M-MuLV Reverse Transcriptase (RNase H-). Second-strand CDNA was synthesized using DNA Polymerase I and RNase H. The remaining overhangs were converted into blunt ends using exonuclease/polymerase activity. After adenylation of the $3^{\prime}$ ends of the DNA fragments, NEBNext Adaptor with a hairpin loop structure was ligated for hybridization. cDNA fragments of 250-300 bp were selected by purifying the library fragments using the AMPure XP system (Beckman Coulter, Beverly, USA). Next, $3 \mu \mathrm{L}$ of the USER Enzyme (NEB, USA) 
was added to the size-selected, adaptor-ligated cDNA, and the mixture was incubated at $37^{\circ} \mathrm{C}$ for 15 min followed by 5 min at $95^{\circ} \mathrm{C}$. Then, PCR was performed with Phusion High-Fidelity DNA polymerase, universal PCR primers, and Index (X) Primer. The PCR products were purified using the AMPure XP system, and library quality was assessed using the Agilent Bioanalyzer 2100 system. Clustering of the index-coded samples was performed using the cBot Cluster Generation System and TruSeq PE Cluster Kit v3-cBot-HS (Illumina), according to the manufacturer's instructions. After cluster generation, the libraries were sequenced using the Illumina Hiseq platform, and paired-end reads were generated.

\subsection{Transcriptome quality control}

Raw data (raw reads) in the FASTQ format were first processed using in-house Perl scripts, and clean data (clean reads) were obtained by removing reads containing the adapter and ploy-N and low-quality reads; simultaneously, Q20, Q30, and GC content of the clean data were calculated. All downstream analyses were performed using high-quality clean data.

\subsection{Transcriptome assembly and gene functional annotation}

To obtain unigenes, transcriptome de novo assembly was performed using Trinity (Grabherr et al., 2011). The assembled unigenes of the transcriptome were used for BLASTx search and annotated against the NCBI non-redundant protein sequence database (NR-NCBI), using an e-value cut-off of 0.00001 . BLASTx alignments (e-value $<0.00001$ ) were then performed between the unigenes and several protein databases, including the Swiss-Prot and Clusters of Orthologous Groups (COG) databases. With NR-NCBI annotation, the Blast2GO program was used to predict gene ontology (GO) terms related to molecular functions, cellular components and biological processes. After obtaining GO annotations for every unigene, we used the GOseq R package and Wallenius noncentral hypergeometric distribution to conduct GO functional classification of all unigenes and to understand the overall distribution of gene functions in this species. We used KOBAS software to test the statistical enrichment of differentially expressed genes among KEGG pathways for the assigned carp orthologs. KEGG orthology (KO) assignments were performed based on the bi-directional best hit $(\mathrm{BHH})$ of the BLAST.

\subsection{Differential expression analysis}

Gene expression levels in each sample were estimated using RSEM (Dewey and Li, 2011). Differential expression analysis of two groups (three biological replicates per condition) was performed using the DESeq R package (1.18.0). The resulting P values were adjusted using Benjamini and Hochberg's approach for controlling the false discovery rate. Genes with adjusted $P$ value $<0.05$ and $\| \log _{2}$ foldchangel $>1$ were identified as differentially expressed.

\subsection{RT-qPCR analysis}

The total RNA of olfactory epithelium, brain, and gut/stomach tissues were reverse-transcribed into cDNA by using the PrimeScript RT Reagent Kit (TaKaRa, Dalian, China). The primers were designed using Primer 5.0 software (Table 1). $\beta$-actin was used as the internal control, and the reaction mixture contained $2 \mu \mathrm{L}$ cDNA, $1 \mu \mathrm{L}$ each of forward and reverse primers, $10 \mu \mathrm{L}$ SYBR Green I Master Mix (TaKaRa), and $6 \mu \mathrm{L}$ PCR- 
grade water. RT-qPCR was performed using an Eppendorf PCR machine (Mastercycler ep Realplex, Hamburg, Germany) with one cycle at $95^{\circ} \mathrm{C}$ for 2 min, followed by 40 cycles at $95^{\circ} \mathrm{C}$ for $15 \mathrm{~s}, 58{ }^{\circ} \mathrm{C}$ for $15 \mathrm{~s}$, and $72{ }^{\circ} \mathrm{C}$ for $20 \mathrm{~s}$. Dissociation curve analysis was performed to determine target specificity. The relative expression levels were calculated using the $2^{-\triangle \Delta C T}$ method, and statistical analysis was performed using one-way ANOVA and tukey's multiple comparison test (SPSS, version 16.0). The qPCR was performed in triplicate to confirm the expression patterns. The significantly differentially expressed olfactory-related receptor genes and five appetite-related genes (GHRL, LEP, NPY, AgRP, and POMC) selected from the transcriptome were examined by RT-qPCR.

\section{Results}

\subsection{Behavioral screening used to identify optimal concentration of amino acids}

The four amino acid phagostimulants concentrations produced different olfactory reaction profiles in silver pomfret with respect to movement and feeding responses (Fig. 1). The Asp, Gly, and Cys groups showed the highest feeding and movement responses with C3 (0.1 mol L-1)-with this concentration, the feeding and movement responses were exhibited, respectively, by $76 \pm 13.1$ fish min- 1 and $145.7 \pm 9.6$ fish min-1 in group Asp, $27.7 \pm 4.5$ fish min-1 and $126 \pm 14.5$ fish min-1 in group Gly, and $20.7 \pm 7$ fish min-1 and $60.3 \pm 9.7$ fish min-1 in group Cys. In the Bet group, the $1.4 \%$ level showed the highest attractant effect in the two responses. Thus, the concentrations $0.1 \mathrm{~mol} \mathrm{L-1}$ (C3) of the amino acids and $1.4 \%$ of Bet were chosen as the optimal exposure dose for all subsequent stimulation and sequencing experiments. In addition, the fish in the negative control group did not show any obvious responses to the fresh water.

To investigate the effect of diet supplemented with $0.1 \mathrm{~mol} \mathrm{L-1}$ of amino acids, we fed the fish four types of diets: (1) Control: without amino acid supplementation, (2) with Bet supplementation, (3) with Gly supplementation, (4) with Asp supplementation, and (5) with Cys supplementation. Compared with the Control, the amino acid groups, especially the Asp and Cys groups, showed significantly shorter ingestion time $(P<0.05)$ (Fig. 2).

\subsection{Transcriptome sequencing and assembly}

A total of 272596054 raw reads were generated, and 260816056 clean reads (95.70\%) were obtained. The mean Q30 and GC content were $97.74 \%$ and $94.48 \%$, respectively (Table S2). A total of 225296 unigenes -which ranged from 201 to $19270 \mathrm{bp}$ in size-were detected in five libraries. The length distribution revealed that most of the unigenes ( $96433 ; 42.80 \%$ ) were in the size range of $201-500 \mathrm{bp}$, followed by those in the size range 500-1000 bp (61 007 unigenes; 27.08\%) and 1-2 kbp (39 357 unigenes; $17.47 \%)$.

\subsection{Gene functional annotation}

The unigenes were annotated by alignment with public protein databases such as NR-NCBI, Swiss-Prot, KEGG, COG, and GO (Table 2). 
The E-value distribution of the top matches showed that $70.9 \%$ of the NR-mapped sequences were in the range of 0 to $1 \mathrm{e}^{-30}$, and $61.5 \%$ had high E-value scores (E-value $<1 \mathrm{e}^{-45}$; Fig. S1A). These results reflect the validity and reliability of our de novo assembly. A substantial majority (94.6\%) of the NR-annotated sequences exhibited $60-100 \%$ similarity to known sequences (Fig. S1B). Additionally, we compared the unigenes to sequences from other fish species; $33.8 \%$ unigenes were best matched to sequences from Larimichthys crocea, followed by those from Stegastes partitus (23.3\%), Oreochromis niloticus (5.2\%), Notothenia coriiceps (3.6\%), Maylandia zebra (2.7\%), and others (31.4\%; Fig.S1C).

The 68085 unigenes were classified into 2675 functional groups of three main categories of GO classifications, which are biological processes, cellular components, and molecular functions (Fig. S2). For biological processes, cellular process (GO:0009987), single-organism process (G0:0044699), and metabolic process (GO:0008152) were highly represented. For cellular components, cell (GO:0005623), followed by cell part (G0:0044464), was highly represented. Finally, the most well-represented molecular functions were binding (GO: 0005488) and catalytic activity (G0:0003824).

Next, 31240 unigenes were annotated and classified into 26 categories (Fig. S3. Signal transduction mechanisms $(5874 ; 18.8 \%)$ was the largest group, followed by general function prediction only (4811; 15.4\%) and posttranslational modification, protein turnover, chaperones (3172; 10.15\%). In addition, 1991 unigenes $(6.37 \%)$ were classified as function unknown, and only six unigenes were assigned to the unnamed protein classification. The KEGG pathway analysis identified the functions and interactions of the unigenes. A total of 41971 unigenes were mapped to 232 pathways in five main categories: cellular processes, environmental information processing, genetic information processing, metabolism, and organismal systems (Fig. S4). In the five main categories, most annotated genes were associated with the PI3K-Akt signaling pathway (ko04151;3.28\%), followed by those associated with pathways related to Endocytosis (ko04144; 3.06\%) and MAPK signaling pathway (ko04010; 2.84\%).

\subsection{Analysis of differentially expressed genes}

A total of 18837 differentially expressed genes (DEGs) were detected, including 15393 upregulated and 3444 downregulated genes between the amino acid groups and control group (Asp vs. Control, Bet vs. Control, Gly vs. Control and Cys vs. Control): 4344 DEGs (3476 upregulated and 868 downregulated) in the Asp group, 5044 DEGs (4158 upregulated and 886 downregulated) in the Gly group, 5759 DEGs (4782 upregulated and 977 downregulated) in the Cys group, and 3690 DEGs (2977 upregulated and 713 downregulated) in the Bet group. Additionally, 34 olfactory-related receptor genes including OR, TAAR, and VR genes were identified (Table 3), and the significant DEGs among groups were further analyzed using RT-qPCR.

\subsection{Gene ontology and KEGG analyses of differentially expressed genes}

According to the GO analysis, a total of 15188 DEGs were mapped to terms in the GO database. Ten highly enriched categories of GO terms $(P$ value $<0.05)$ are listed in Table S3. Some of the the terms were 
intracellular ribonucleoprotein complex (G0:0030529) and ribonucleoprotein complex (GO:1990904) were highly enriched in all four groups.

According to the KEGG pathway analysis, 11398 DEGs were mapped to the KEGG database. Ten highly enriched categories of KEGG ( $P$ value $<0.05)$ are listed in Table S4. Some of the highly enriched categories were ribosome (ko03010), neurotrophin signaling pathway (ko04722) and thyroid hormone signaling pathway (ko04919).

\subsection{RT-qPCR analysis of olfactory-related genes}

We selected four significantly differentially expressed olfactory-related receptor genes - OR 7A17 (C103772), OR 4K1 (C80459), TAAR 13C (C81603), and V2R 1 (C78258)-for the qPCR analysis. The results were mostly consistent with those of the RNA-seq analysis (Fig. 3). For, The expression levels of $O R 7 A 17$ and $O R 4 K 1$ were significantly lower in the Cys group than in the other amino acid groups, whereas the expression levels of TAAR $13 C$ and $V 2 R 1$ were significantly higher in the Asp group than in the other amino acid groups.

$N P Y$ and $A g R P$ in the brain and GHRL in the gut/stomach were upregulated, but $P O M C$ in the brain and $L E P$ in the gut/stomach were downregulated (Fig. 4). The expression levels of $A g R P$ were significantly higher in the Asp and Gly groups than in the other groups; the expression levels of $N P Y$ were significantly higher in the Asp and Bet groups than in the other groups; and the expression levels of POMC in the Asp and Gly groups were significantly lower than those in control and the Cys group but were similar to those in the Bet group. The expression levels of $L E P$ were significantly lower in the Cys group than in the other groups, whereas the expression levels of GHRL were significantly higher in the Asp and Cys groups than in the other groups.

\section{Discussion}

\subsection{Effect of amino acids on fish olfactory behavior and food intake}

Amino acids can stimulate the olfactory receptor neurons of fish and produce the electrical olfactory signal (Sato, 2001). In the present study, we selected three high-content amino acids in jellyfish Rhopilema esculentum (Yu et al., 2014, Xiguang, 2004), and the silver pomfret showed a high preference for them. Silver pomfret show a high feeding preference for jellyfish (Liu et al., 2015); therefore, it was suggested that the amino acids may be one type of main attractant substances in jellyfish, leading to the strong appetite of silver pomfret for them. Many previous researchers have studied olfactory stimulants in fish by detecting the electrical olfactory signals through electroolfactograms (EOGs) (Meredith et al., 2012, Ishida and Kobayashi, 2006). In the present study, we assessed the behaviors of fish upon stimulation with the three amino acids and divided them into two kinds of responses (feeding and movement) to simulate the natural predation process (detection and ingestion) so that the fish could indicate the direct response to stimuli (preference or avoidance). We found that the silver pomfret did not show significant difference responses between $0.1 \mathrm{~mol}$ L-1 and higher concentrations of amino acids, 
and it has been reported that the olfactory receptors of fish have a threshold for amino acids (Ishida and Kobayashi, 2006, Zhao, 2007); therefore, we speculated that the production of an olfactory signal in fish is regulated once the concentration of stimulus achieves a certain threshold, which prevents an unrestricted increase in the olfactory signal to protect the brain.

\section{2 olfactory-related receptors for amino acids in silver pomfret}

Amino acids can bind to olfactory-related receptors that activate the $G$ protein to transfer the olfactory signal (Saberi and Seyed-allaei, 2016, Ronnett and Moon, 2002). In the present study, OR, VR, and TAAR, three olfactory receptor gene families in fish, were all observed to be differentially expressed, which was consistent with our previous study (Hu et al., 2017). We suggested that a single amino acid could stimulate the three receptors which may work synergistically for the production of olfactory signal. Main olfactory receptor (OR) is the largest gene family among three olfactory-related receptor families, and these receptors play an important role in olfactory activity (Alioto and Ngai, 2006, Saraiva and Korsching, 2007). We also obtained similar results indicating that six types of OR genes in silver pomfret were considerably more differentially expressed when the fish were exposed to the four phagostimulants than TAAR and VR genes, which suggested that $O R$ is the main receptor of amino acids. A previous study has shown that the TAAR genes could be activated by trace levels of amines (Borowsky et al., 2001); thus, dilution of amino acids in culturing water in the present study may have achieved the trace levels, which led to the differential expression of TAAR genes. Meanwhile, the VR genes were upregulated after exposure to amino acids, which was similar to findings of other studies (Hansen et al., 2003, Sato, 2001). Because VRs have been reported to be pheromone receptors (Muramoto et al., 2011), we speculate that the three amino acids-Asp, Cys, Gly-might be the partial components of pheromones in fish or stimulate pheromone release in fish. Furthermore, some studies have identified a fourth type of olfactory-related receptor for formylated peptides, termed FPR, expressed in mammals (Rivière et al., 2009). However, we did not identity this gene family in the olfactory epithelium transcriptome of silver pomfret, and there are no reports about this receptor gene in other fishes such as large yellow croaker (Ao et al., 2015). From the aspect of evolution, the absence of this gene family in fish may be attributable to the water solubility of formylated peptides.

\subsection{Regulation of fish appetite through olfactory}

After olfactory stimulation with amino acids, five appetite-related genes including $N P Y, A g R P$, and $P O M C$ (expressed in the whole brain) and $L E P$ and GHRL (expressed in the gut and stomach) were examined using RT-qPCR. In the central nervous system, the hormones NPY, AgRP, and POMC are produced in the hypothalamus and transported to target sites though blood (Bertucci et al., 2019). In the present study, NPY, AgRP, and POMC were all significantly differentially expressed in the brains of fish exposed to phagostimulants. NPY and AgRP improve the fish feed intake, but POMC has the opposite effect (Volkoff, 2016); thus, upregulated NPY and AgRP and downregulated POMC may promote appetite in silver pomfret. Additionally, we found that $L E P$ was downregulated, but GHRL was upregulated in the gut and stomach. In the adipocytokine signaling pathway (map04920), leptin activates POMC and suppresses the 
AMPK signaling, which leads to reduced expression of NPY and AgRP through the JAK-STAT signaling pathway (map04630) (Minokoshi et al., 2004). The target sites of GHRL, an orexigenic factor, are located in the hypothalamus and pituitary (Massadi et al., 2011, Matsuda et al., 2006). Thus, the results indicated that these two key appetite-regulating factors are secreted by the gut and stomach of fish even without nutrient stimulation, and they exert their anorectic (leptin) or orexigenic (GHRL) effects by modulating the expression of neuropeptides in the brain.

The feed intake of fish could influence the appetite though neuroendocrine signaling (homeostatic regulation by the peripheral and central nervous systems) (Bertucci et al., 2019). In the present study, amino acids diluted in water induced the feeding behavior in fish (biting the air stone) through olfactory stimulation, which indicated that olfactory also regulates the appetite through the central and peripheral nervous systems. We detected many DEGs of olfactory-related receptors in the phagostimulant test groups. The activation of olfactory-related receptors could lead to the production of electrical olfactory signals by olfactory transduction (Munger et al., 2001, Hu et al., 2019). It has been reported that the brain and the gut can interact with each other through hormones and neurons (Kaelberer et al., 2018).

Therefore, the electrical olfactory signal transmitted to the brain may be able to regulate the appetiterelated hormones of fish through the OE-brain-gut/stomach-axis neurons, and the hormones in gut and stomach also feed back to the brain, through which appetite is induced by the combined action of the hormones.

We found that Asp could effectively stimulate the olfactory-related receptors of silver pomfret, leading to the significant differential expression of olfactory-related genes. The feeding behavior and the expression patterns of five appetite-related genes of silver pomfret suggest that Asp significantly improves appetite and feeding performance, indicating that it is a suitable phagostimulant to partly replace the jellyfish in silver pomfret diet.

\section{Conclusion}

In the present study, we selected three types of high-content amino acids in jellyfish to stimulate the olfactory-related receptors of silver pomfret. The fish showed significant attractant responses to these amino acids, which were examined on the basis of feed ingestion behaviors. Transcriptomics of the olfactory epithelium indicated that many olfactory-related receptor genes were differentially expressed after exposure to aspartic acid, glycine, and cysteine. Moreover, the expression levels of five appetiterelated genes were significantly changed in the brain and gut/stomach. Thus, olfactory-related receptors induced by amino acids might regulate appetite in silver pomfret through the olfactory epithelium-braingut/stomach axis. Using these data, we identified effective amino acid phagostimulants which could be supplied and partly replace the jellyfish in silver pomfret diet. Furthermore, the results have improved our understanding of the mechanism of olfactory-related receptors regulating appetite in fish.

\section{Abbreviations}

$\mathrm{OE}$ 
olfactory epithelium; ORs:olfactory receptors; VRs:vomeronasal receptors; TAARs:trace amine-associated receptors; NPY:neuropeptide Y; AgRP:agouti-related protein; POMC:proopiomelanocortin; GHRL:ghrelin; LEP:leptin; Asp:L-Aspartic acid; Gly:L-Glycine; Cys:L-Cysteine; Bet:betaine ; NR-NCBI:the NCBI nonredundant protein sequence database ; COG:Clusters of Orthologous Groups ; GO:gene ontology; DEGs:differentially expressed genes; EOGs:electroolfactograms

\section{Declarations}

\section{Ethics approval and consent to participate}

The silver pomfret in our study were bred and cultured by ourselves and were sampled after anaesthesia with MS222. All fish experiments were conducted in accordance with the recommendations of the National Institutes of Health Guide for the Care and Use of Laboratory Animals. The Animal Care and Use Committee of Ningbo University approved the protocols.

\section{Consent for publication}

Not applicable

\section{Availability of data and materials}

The datasets (raw reads) analysed in this study were uploaded to NCBI' s Sequence Read Archive (BioProject accession numbers: PRJNA597448;https://dataview.ncbi.nlm.nih.gov/object/PRJNA597448).

\section{Competing interests}

The authors declare no conflict of interest.

\section{Funding}

This work was supported by the Natural Science Foundation of China (31772869 and 31872586), the Natural Science Foundation of Zhejiang (LY18C190008 and LY18C1900013), the Agriculture Key Special Project of Ningbo (2015C110003), the Zhejiang Major Science Project (2019C02059), the Optimization and Utilization of Acute Toxicity Test Methods for Luminescent Bacteria (2019ZS02) and the K.C. Wong Magna Fund in Ningbo University, Li Dak Sum Yip Yio Chin Kenneth Li Marine Biopharmaceutical Development Fund, National 111 Project of China. The funders had no role in study design, data collection and analysis, decision to publish, or preparation of the manuscript.

\section{Authors' contributions}

$\mathrm{JH}, \mathrm{QL}, \mathrm{XY}$ and $\mathrm{YW}$ designed and supervised the project. SK, MZ developed and implemented the experiment. $\mathrm{YZ}, \mathrm{WG}$ and $\mathrm{YS}$ performed the analysis. JH and $\mathrm{YL}$ prepared the manuscript. All authors read and approved the final manuscript. 


\section{Acknowledgements}

Not applicable

\section{Authors' information}

${ }^{a}$ Key Laboratory of Applied Marine Biotechnology, Ningbo University, Ministry of Education, Ningbo, China; ${ }^{b}$ Key Laboratory of Marine Biotechnology of Zhejiang Province, Ningbo University, Ningbo, China; ${ }^{c}$ College of marine Sciences, Ningbo University, Ningbo, China; ${ }^{d}$ Ningbo Entry-Exit Inspection and Quarantine Bureau Technical Center, Ningbo, China; ${ }^{e}$ Ningbo Marine and Fishery Research Institute, Ningbo, China.

\section{References}

1. Alioto, T. S., \& Ngai, J. (2006). The repertoire of olfactory C family G protein-coupled receptors in zebrafish: candidate chemosensory receptors for amino acids. Bmc Genomics, 7(1), 309.

2. Ao, J., Mu, Y., Xiang, L., Fan, D., Feng, M., Zhang, S., \& Ding, Y. (2015). Genome sequencing of the perciform fish Larimichthys crocea provides insights into molecular and genetic mechanisms of stress adaptation. Science Foundation in China, 11(3), 8-8.

3. Asanomiyoahi, M., Suda, T., Yasuoka, A., Osima, S., Yamashita, S., \& Abe, K. (2000). Random expression of main and vomeronasal olfactory receptor genes in immature and mature olfactory epithelia of Fugu rubripes. Journal of Biochemistry, 127(5), 915-924.

4. Bell, E. A., Perera, K. P. W. C., Nunn, P. B., Simmonds, M. S. J., \& Blaney, W. M. (1996). Non-protein amino acids of Lathyrus latifolius as feeding deterrents and phagostimulants in Spodoptera littoralis. Phytochemistry, 43(5), 1003-1007.

5. Bertucci, J. I., Blanco, A. M., Sundarrajan, L., Rajeswari, J. J., Velasco, C., \& Unniappan, S. (2019). Nutrient regulation of endocrine factors influencing feeding and growth in fish. Frontiers in endocrinology, 10, 83-83. doi: 10.3389/fendo.2019.00083

6. Borowsky, B., Adham, N., Jones, K. A., Raddatz, R., Artymyshyn, R., Ogozalek, K. L., \& Pathirana, S. (2001). Trace amines: identification of a family of mammalian $G$ protein-coupled receptors. Proceedings of the National Academy of Sciences of the United States of America, 98(16), 89668971. doi: 10.1073/pnas.151105198

7. Buck, L., \& Axel, R. (1991). A novel multigene family may encode odorant receptors: a molecular basis for odor recognition. Cell, 65(1), 175-187. doi: 10.1016/0092-8674(91)90418-X

8. Campos, V. F., Collares, T., Deschamps, J. o. C., Seixas, F. K., Dellagostin, O. A., Lanes, C. F. C., \& Sampaio, L. A. (2010). Identification, tissue distribution and evaluation of brain neuropeptide $Y$ gene expression in the Brazilian flounder Paralichthys orbignyanus. Journal of Biosciences, 35(3), 405413.

9. Cao, Y., Oh, B. C., \& Stryer, L. (1998). Cloning and localization of two multigene receptor families ingoldfish olfactory epithelium. Proceedings of the National Academy of Sciences of the United 
States of America, 95(20), 11987-11992. doi: 10.1073/pnas.95.20.11987

10. Cummings, D. E., Weigle, D. S., Frayo, R. S., Breen, P. A., Ma, M. K., Dellinger, E. P., \& Purnell, J. Q. (2002). Plasma ghrelin levels after diet-Induced weight loss or gastric bypass surgery. New English Journal of Medicine, 346(21), 1623-1630.

11. Dewey, C. N., \& Li, B. (2011). RSEM: accurate transcript quantification from RNA-Seq data with or without a reference genome. Bmc Bioinformatics, 12(1), 323-323.

12. Franklyn, A., Wang, Y., Yu, N., Wang, J., \& Le, Q. (2017). Transcriptome analysis reveals L-amino acids as olfactory stimulant in the large yellow croaker (Larimichthys crocea). Journal of Aquaculture Research and Development, 8(475), 2.

13. Gloriam, D. E., Bjarnadóttir, T. K., Yan, Y. L., Postlethwait, J. H., Schiöth, H. B., \& Fredriksson, R. (2005). The repertoire of trace amine G-protein-coupled receptors: large expansion in zebrafish. Molecular Phylogenetics \& Evolution, 35(2), 470-482. doi: 10.1016/j.ympev.2004.12.003

14. Gong, N., Jönsson, E., \& Bjornsson, B. T. (2015). Acute anorexigenic action of leptin in rainbow trout is mediated by the hypothalamic Pi3k pathway. Journal of Molecular Endocrinology, 56(3), $227-238$.

15. Grabherr, M. G., Haas, B. J., Yassour, M., Levin, J. Z., Thompson, D. A., Amit, I., \& Zeng, Q. (2011). Fulllength transcriptome assembly from RNA-Seq data without a reference genome. Nature biotechnology, 29(7), 644.

16. Hansen, A., Rolen, S. H., Anderson, K., Morita, Y., Caprio, J., \& Finger, T. E. (2003). Correlation between olfactory receptor cell type and function in the channel catfish. Journal of Neuroscience the Official Journal of the Society for Neuroscience, 23(28), 9328-9339.

17. Hara, T. J. (2010). Feeding behaviour in some teleosts is triggered by single amino acids primarily through olfaction. Journal of Fish Biology, 68(3), 810-825.

18. Helene, V. (2016). The neuroendocrine regulation of food Intake in fish: a review of current knowledge. Frontiers in Neuroscience, 10.

19. Hu, J., Wang, Y., Le, Q., Na, Y., Cao, X., Zheng, H., \& Wu, X. (2017). Transcriptomic analysis reveals olfactory-related genes expression in large yellow croaker (Larimichthys crocea) regulated by taurine: May be a good phagostimulant for all-plant protein diets. Aquaculture Research, 49(2).

20. Hu, J., Wang, Y., Le, Q., Yu, N., Cao, X., Kuang, S., \& Yang, Y. (2019). Transcriptome sequencing of olfactory-related genes in olfactory transduction of large yellow croaker (Larimichthy crocea) in response to bile salts. PeerJ, 7, e6627.

21. Ishida, Y., \& Kobayashi, H. (2006). Stimulatory effectiveness of amino acids on the olfactory response in an algivorous marine teleost, the rabbitfish Siganus fuscescens Houttuyn. Journal of Fish Biology, 41(5), 737-748.

22. Johnstone, K. A., Lubieniecki, K. P., Koop, B. F., \& Davidson, W. S. (2012). Identification of olfactory receptor genes in Atlantic salmon Salmo salar. Journal of Fish Biology, 81(2), 559-575. doi: 10.1111/j.1095-8649.2012.03368.x

23. Kaelberer, M. M., Buchanan, K. L., Klein, M. E., Barth, B. B., Montoya, M., Shen, X., \& Bohorquez, D. V. (2018). A gut-brain neural circuit for nutrient sensory transduction. Science, 361(6408). 
24. KAUPP, U. B. 2010. Olfactory signalling in vertebrates and insects: differences and commonalities. Nature Reviews Neuroscience, 11, 188-200.

25. Koide, T., Miyasaka, N., Morimoto, K., Asakawa, K., Urasaki, A., Kawakami, K., \& Yoshihara, Y. (2009). Olfactory neural circuitry for attraction to amino acids revealed by transposon-mediated gene trap approach in zebrafish. Proceedings of the National Academy of Sciences of the United States of America, 106(24), 9884-9889.

26. Kolmakov, N. N., Kube, M., Reinhardt, R., \& Av., C. (2008). Analysis of the goldfish Carassius auratus olfactory epithelium transcriptome reveals the presence of numerous non-olfactory GPCR and putative receptors for progestin pheromones. Bmc Genomics, 9(1), : 429. doi: 10.1186/1471-2164-9429

27. Liberles, S. D., \& Buck, L. B. (2006). A second class of chemosensory receptors in the olfactory epithelium. Nature, 442(7103), 645-650. doi: 10.1038/nature05066

28. Liu, C. S., Chen, S. Q., Zhuang, Z. M., Yan, J. P., Liu, C. L., \& Cui, H. T. (2015). Potential of utilizing jellyfish as food in culturing Pampus argenteus juveniles. Hydrobiologia, 754(1), 189-200.

29. Mackenzie, D. S., Vanputte, C. M., \& Leiner, K. A. (1998). Nutrient regulation of endocrine function in fish. Aquaculture, 161(1), 3-25.

30. Massadi, O. A., TschöpJ, M. H., \& Tong, J. (2011). Ghrelin acylation and metabolic control. Peptides, 32(11), 0-2308.

31. Matsuda, K., Miura, T., Kaiya, H., Maruyama, K., Shimakura, S.-I., Uchiyama, M., \& Shioda, S. (2006). Regulation of food intake by acyl and des-acyl ghrelins in the goldfish. Peptides, 27(9), 0-2325.

32. Meredith, T. L., Caprio, J., \& Kajiura, S. M. (2012). Sensitivity and specificity of the olfactory epithelia of two elasmobranch species to bile salts. The Journal of Experimental Biology, 215(15), 2660-2667.

33. Miguel, C.-R. J., \& Ector, P. R. (2003). Endogenous melanocortin antagonist in fish: structure, brain mapping, and regulation by fasting of the goldfish agouti-related protein gene. Endocrinology, 144(10), 4552-4561.

34. Minokoshi, Y., Alquier, T., Furukawa, N., Kim, Y.-B., Lee, A., Xue, B., \& Birnbaum, M. J. (2004). AMPkinase regulates food intake by responding to hormonal and nutrient signals in the hypothalamus. Nature, 428(6982), 569.

35. Munger, S. D., Lane, A. P., Zhong, H., Leinders-Zufall, T., Yau, K.-W., Zufall, F., \& Reed, R. R. (2001). Central role of the CNGA4 channel subunit in Ca2+-calmodulin-dependent odor adaptation. Science, 294(5549), 2172-2175.

36. Muramoto, K., Hagino-Yamagishi, K., Tonosaki, K., \& Kaba, H. (2011). Accessory olfactory bulb neurons are required for maintenance but not induction of V2R vomeronasal receptor gene expression in vitro. Neuroscience Letters, 500(1), 6-9. doi: 10.1016/j.neulet.2011.05.232

37. Niimura, Y. (2009). On the origin and evolution of vertebrate olfactory receptor genes: comparative genome analysis among 23 Chordate Species. Genome Biology \& Evolution, 1(1), 34-44. doi: 10.1093/gbe/evp003 
38. Pfister, P., \& Rodriguez, I. (2005). Olfactory expression of a single and highly variable V1r pheromone receptor-like gene in fish Species. Proceedings of the National Academy of Sciences of the United States of America, 102(15), 5489-5494. doi: 10.1073/pnas.0402581102

39. Rivière, S., Challet, L., Fluegge, D., Spehr, M., \& Rodriguez, I. (2009). Formyl peptide receptor-like proteins are a novel family of vomeronasal chemosensors. Nature, 459(7246), 574-577. doi: $10.1038 /$ nature08029

40. Ronnett, G. V., \& Moon, C. (2002). G proteins and olfactory signal transduction. Annual review of physiology, 64(1), 189-222.

41. Saberi, M., \& Seyed-allaei, H. (2016). Odorant receptors of Drosophila are sensitive to the molecular volume of odorants. Scientific Reports, 6(1), 25103. doi: 10.1038/srep25103

42. Saraiva, L. R., \& Korsching, S. I. (2007). A novel olfactory receptor gene family in teleost fish. Genome Research, 17(10), 1448-1457. doi: 10.1101/gr.6553207

43. Sato, K., and Noriyo Suzuki. (2001). Whole-cell Response Characteristics of Ciliated and Microvillous Olfactory Receptor Neurons to Amino Acids, Pheromone Candidates and Urine in Rainbow Trout. Chemical senses, 26(9), 1145-1156.

44. Si, Z., Mengwei, Y., Jilin, X., Chunyang, G., Huakun, Z., Jiabao, H., .. Xiaojun, Y. (2015). Lipid Profile in Different Parts of Edible Jellyfish Rhopilema esculentum. Journal of Agricultural \& Food Chemistry, 63(37), 8283-8291.

45. Tandler, A., Berg, B. A., Kissil, G. W., \& Mackie, A. M. (1982). Effect of food attractants on appetite and growth rate of gilthead bream, Sparus aurata L. Journal of Fish Biology, 20(6), 673-681.

46. Volkoff, H. (2016). The Neuroendocrine Regulation of Food Intake in Fish: A Review of Current Knowledge. Frontiers in Neuroscience, 10, 540.

47. Xiguang, L. (2004). Studies on Chemical Components and Biological Activities of Jellyfish (Rhopilema esculentum Kishinouye). Graduate School of Chinese Academy of Sciences (The Institute of Oceanology).

48. Yu, H., Li, R., Song, L., Xing, R. E., Chen, X., \& Li, P. (2014). Amino acid composition and nutritional quality of gonad from jellyfish Rhopilema esculentum. Biomedicine \& Preventive Nutrition, 4(3), 399402.

49. Zhao, H. Y. (2007). Studies on feeding stimulants for gibel carp (Carassius auratus gibelio). Chinese Academy of Sciences (Institute of Hydrobiology).

\section{Tables}


Table 1

Primers used for real-time quantitative PCR (RT-qPCR)

\begin{tabular}{|lll|}
\hline Gene name & Forward primer sequence & Reverse primer sequence \\
\hline OR $7 A 17$ & TTCGTGAGACCTCCCGTTAT & CGAGCATACCAAGAGCACC \\
\hline OR 4 K 1 & GTGCGTGTCTTGTTCTCCCT & TCTGGCGACGAGTGGTGA \\
\hline V2R 1 & CCGTCATTTGTGAATCCTCC & TCCCTTAAACATAACATTGCGT \\
\hline GHRL & TGCCACAGTCTGAGTGAACA & CAGTCTCAATGCCTTCCAAT \\
\hline LEP & GAAGTCATCACTCCTTCCATTG & ATTCTCACTGCTCTCTCCTCTC \\
\hline NPY & GAGGCGGCTCATCAAGG & GAAAGCACAGACACTCTTCCAC \\
\hline AgRP & TAGTCGCCTGGTCTTCCCT & CTTCTTCCTACTCGCCTTCC \\
\hline POMC & GCTCTCGCCTCCTTATCTCT & GCTCTGTCACCACCTACAATCT \\
\hline$\beta$-actin & TGGCATCACACCTTCTACAAC & ACGACCAGAGGCATACAGG \\
\hline
\end{tabular}

Table 2

Summary of functional annotation of unigenes

\begin{tabular}{|lll|}
\hline Database & Number of Unigenes & Percentage (\%) \\
\hline NR -NCBI & 74459 & 33.04 \\
\hline NT-NCBI & 84466 & 37.49 \\
\hline KO & 41971 & 18.62 \\
\hline Swiss-Prot & 65072 & 28.88 \\
\hline PFAM & 67903 & 30.13 \\
\hline GO & 68085 & 30.22 \\
\hline KOG & 31240 & 13.86 \\
\hline
\end{tabular}


Table 3

Differentially expressed olfactory-related receptor genes in four groups (treated with aspartic acid [Asp], glycine [Gly], cysteine [Cys] and betaine [Bet])

\section{Gene ID $\quad \log _{2}$ (fold change) P value Description}

\section{Asp vs. Control}

C103772 $1.5456 \quad 3.92 \mathrm{E}-05 \quad$ Olfactory receptor 7A17

C80459 $-2.5972 \quad 3.19 \mathrm{E}-05 \quad$ Olfactory receptor 4K1

$\begin{array}{llll}\text { C86326 } & 3.7347 & 0.0013835 & \text { Olfactory receptor family C subfamily } 11 \text { member } 4\end{array}$

C81603 $1.5559 \quad$ 4.34E-05 Trace amine-associated receptor 13c

$\begin{array}{llll}C 97869 & 1.2757 & 0.0029339 & \text { Trace amine-associated receptor } 4\end{array}$

C78258 $1.9923 \quad 2.70 \mathrm{E}-22 \quad$ Vomeronasal type-2 receptor 1

$\begin{array}{llll}\text { C93486 } & -6.0722 & 4.76 \mathrm{E}-06 & \text { Vomeronasal type-2 receptor } 1\end{array}$

\section{Bet vs. Control}

\begin{tabular}{|llll|}
\hline C103772 & 2.6678 & $3.58 \mathrm{E}-14$ & Olfactory receptor 7A17 \\
\hline C45007 & 3.9258 & 0.00493 & Olfactory receptor 5B2 \\
\hline C68391 & 2.3003 & 0.0030762 & Olfactory receptor 7A17 \\
\hline C77820 & 1.8217 & 0.00038692 & Olfactory receptor family C subfamily 11 member 4 \\
\hline C72609 & 1.7466 & $1.32 \mathrm{E}-05$ & Trace amine-associated receptor 4 \\
\hline C81603 & 2.4654 & $3.08 \mathrm{E}-11$ & Trace amine-associated receptor 13c \\
\hline C97869 & 1.4433 & 0.004767 & Trace amine-associated receptor 4 \\
\hline C78258 & 1.9589 & $7.54 \mathrm{E}-17$ & Vomeronasal type-2 receptor 1 \\
\hline
\end{tabular}

Gly vs. Control

\begin{tabular}{llll} 
C103772 & 2.0012 & $8.83 \mathrm{E}-08$ & Olfactory receptor 7A17 \\
\hline C58387 & 4.3835 & 0.0048477 & Olfactory receptor 1 \\
\hline C80459 & -1.8914 & 0.0021702 & Olfactory receptor 4K1 \\
\hline C72609 & 1.4472 & 0.00018782 & Trace amine-associated receptor 4 \\
\hline C81603 & 2.2074 & $1.52 \mathrm{E}-09$ & Trace amine-associated receptor 13c \\
\hline C97869 & 1.508 & 0.00076369 & Trace amine-associated receptor 4 \\
\hline C78258 & 1.7824 & $2.55 \mathrm{E}-15$ & Vomeronasal type-2 receptor 1 \\
\hline C93486 & 1.8666 & $6.41 \mathrm{E}-16$ & Vomeronasal type-2 receptor 1 \\
\hline
\end{tabular}




\begin{tabular}{|llll|}
\hline Gene ID & Log $_{2}$ (fold change) & P value & Description \\
\hline \multicolumn{2}{|l}{ Cys vs. Control } & & \\
\hline C103772 & 2.2284 & $2.41 \mathrm{E}-11$ & Olfactory receptor 7A17 \\
\hline C58387 & 4.6617 & 0.00072462 & Olfactory receptor 1 \\
\hline Cl68391 & 1.9925 & 0.0038765 & Olfactory receptor 7A17 \\
\hline C80459 & -2.1562 & 0.00055356 & Olfactory receptor 4K1 \\
\hline C72609 & 1.4772 & $2.12 \mathrm{E}-05$ & Trace amine-associated receptor 4 \\
\hline C81603 & 2.3492 & $1.43 \mathrm{E}-12$ & Trace amine-associated receptor 13c \\
\hline C95324 & 1.9513 & 0.0014775 & Trace amine-associated receptor 1 \\
\hline C97869 & 1.4476 & 0.00036591 & Trace amine-associated receptor 4 \\
\hline C77261 & 2.9141 & 0.0022176 & Vomeronasal type-1 receptor 1 \\
\hline C78258 & 2.1357 & $1.67 \mathrm{E}-26$ & Vomeronasal type-2 receptor 1 \\
\hline C93486 & 1.5948 & $3.97 \mathrm{E}-13$ & Vomeronasal type-2 receptor 1 \\
\hline
\end{tabular}

\section{Figures}


Feeding Response

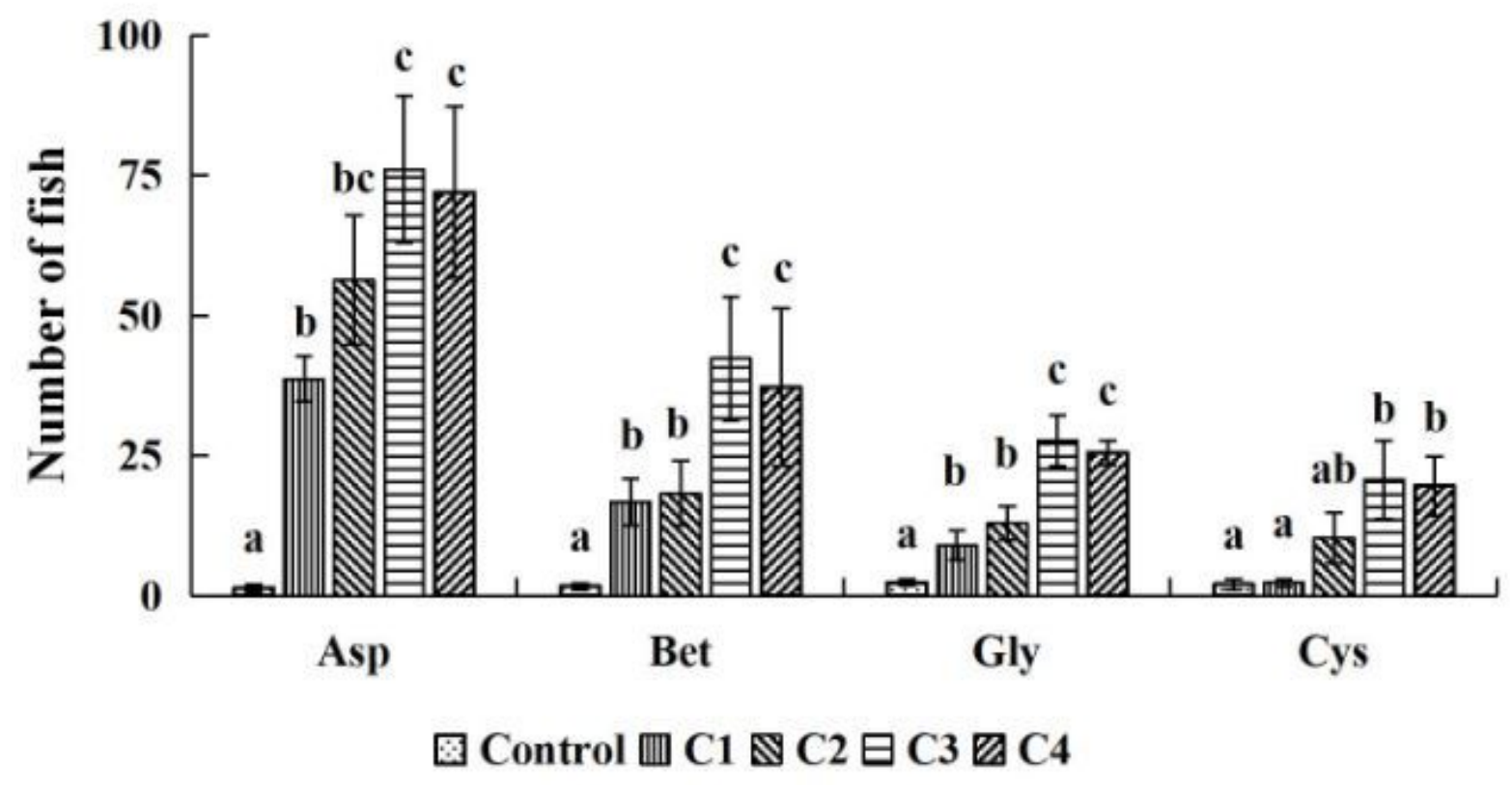

Movement Response

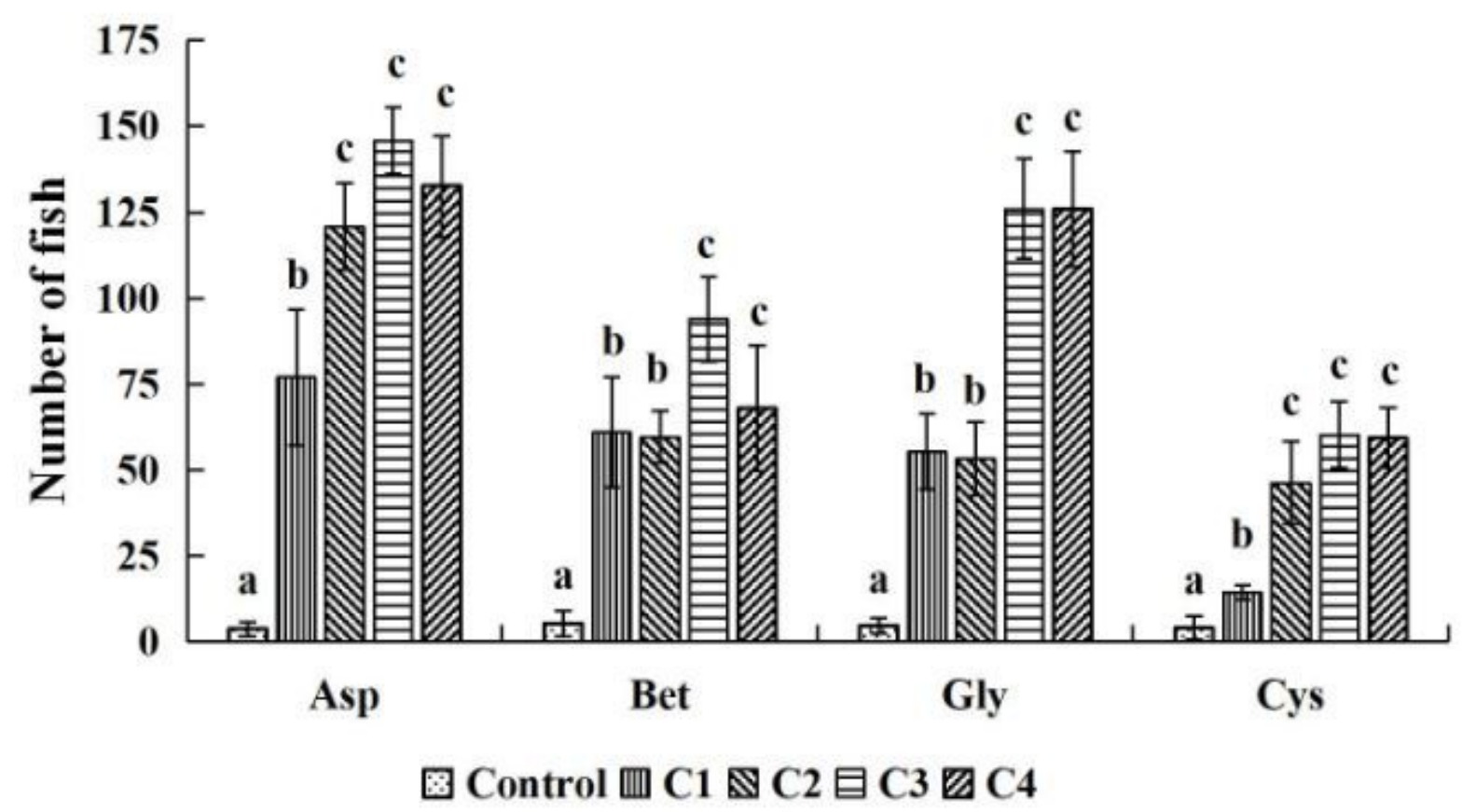

Figure 1

Responses of silver pomfret to increasing concentrations of the amino acids aspartic acid (Asp), glycine (Gly), and cysteine (Cys) and positive control betaine (Bet). The Y-axis shows the number of fish exhibiting the behavioral response in $1 \mathrm{~min}$ (average for $5 \mathrm{~min}$ ); C1/2/3/4: Concentration 1/2/3/4 for amino acids and positive control; Values are means \pm standard deviations $(n=9) ; P<0.05$. 


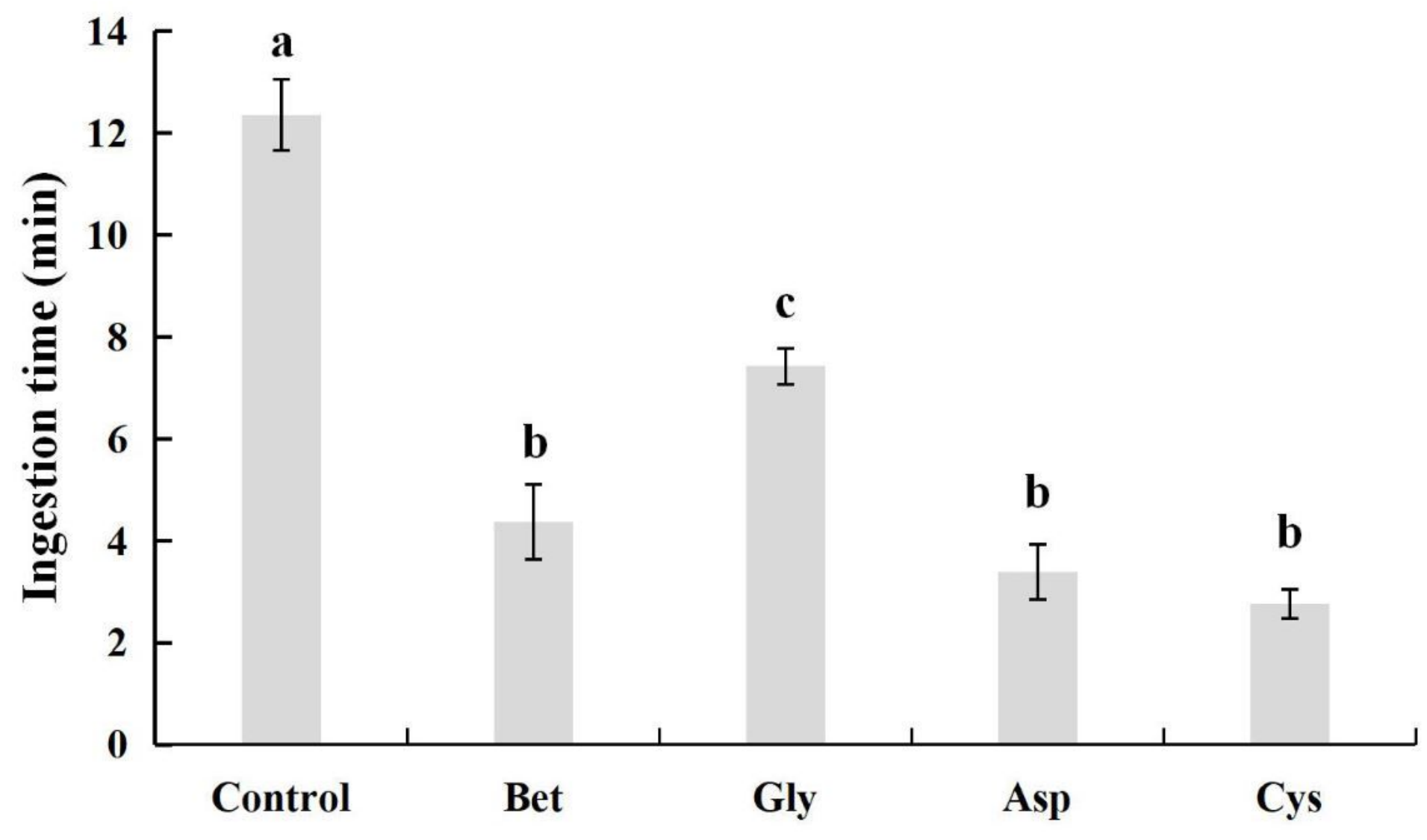

Figure 2

Ingestion time of silver pomfret fed with five kinds of diets. Values are means \pm standard deviations $(n=9)$; $\mathrm{P}<0.05$. 

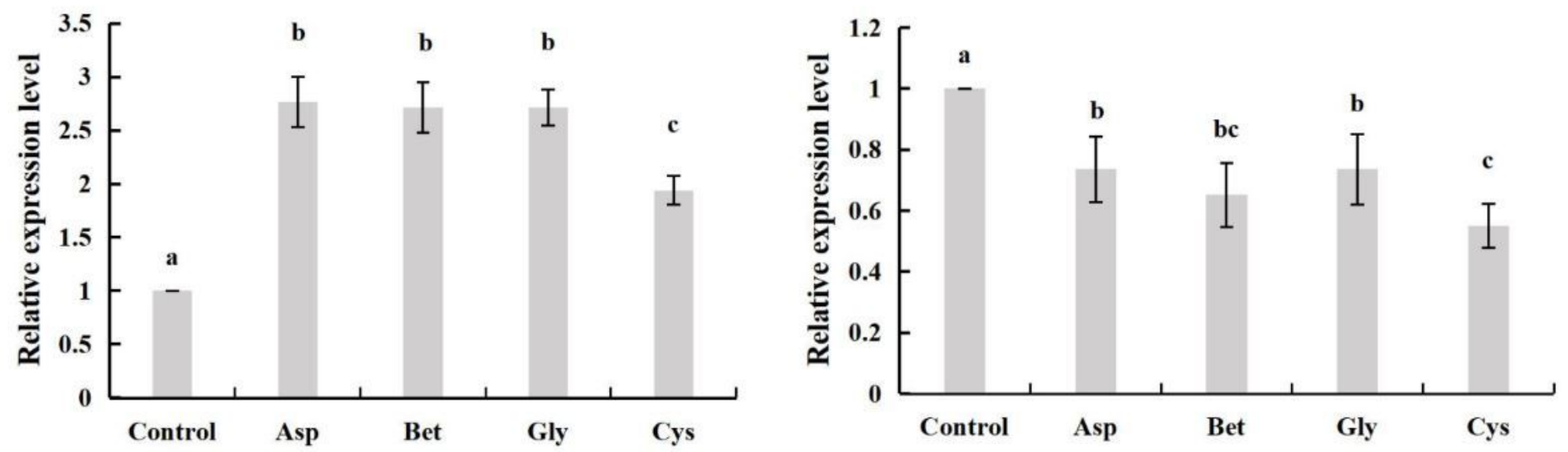

TAAR $13 C$

$\operatorname{V2R} 1$
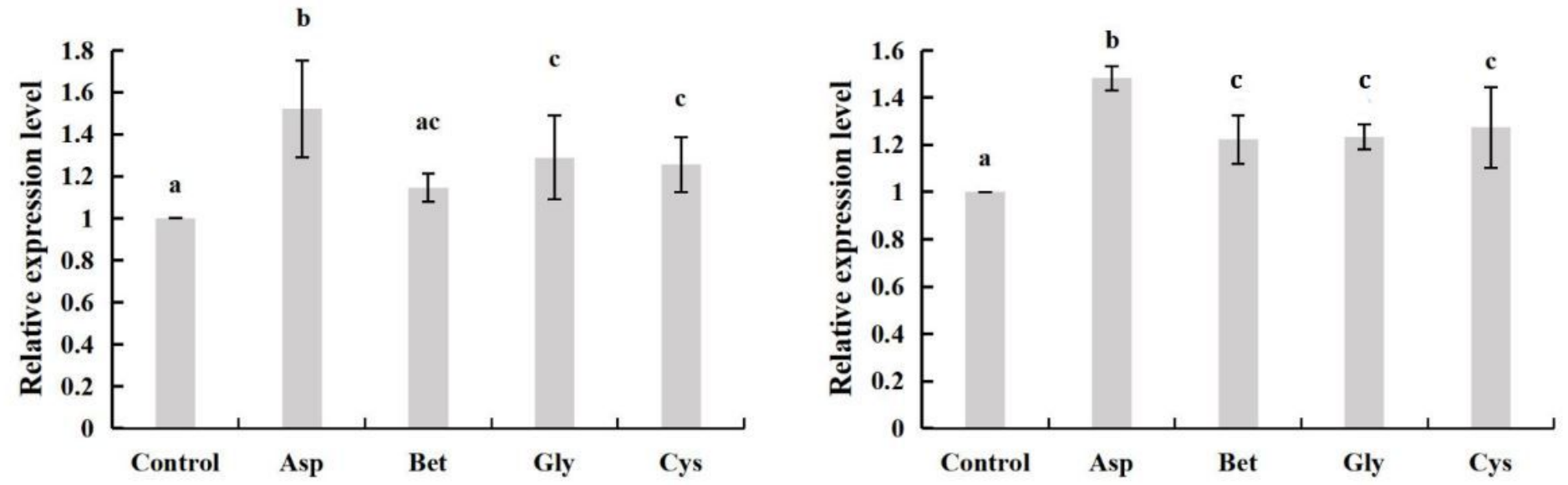

Figure 3

Expression of olfactory-related genes in olfactory transduction of silver pomfret exposed to the amino acids aspartic acid (Asp), glycine (Gly), and cysteine (Cys) and positive control betaine (Bet). The results were calculated according to the $2-\square C C T$ method using $\beta$-actin as an internal reference gene; Values are means \pm standard deviations $(n=3) ; P<0.05$. 

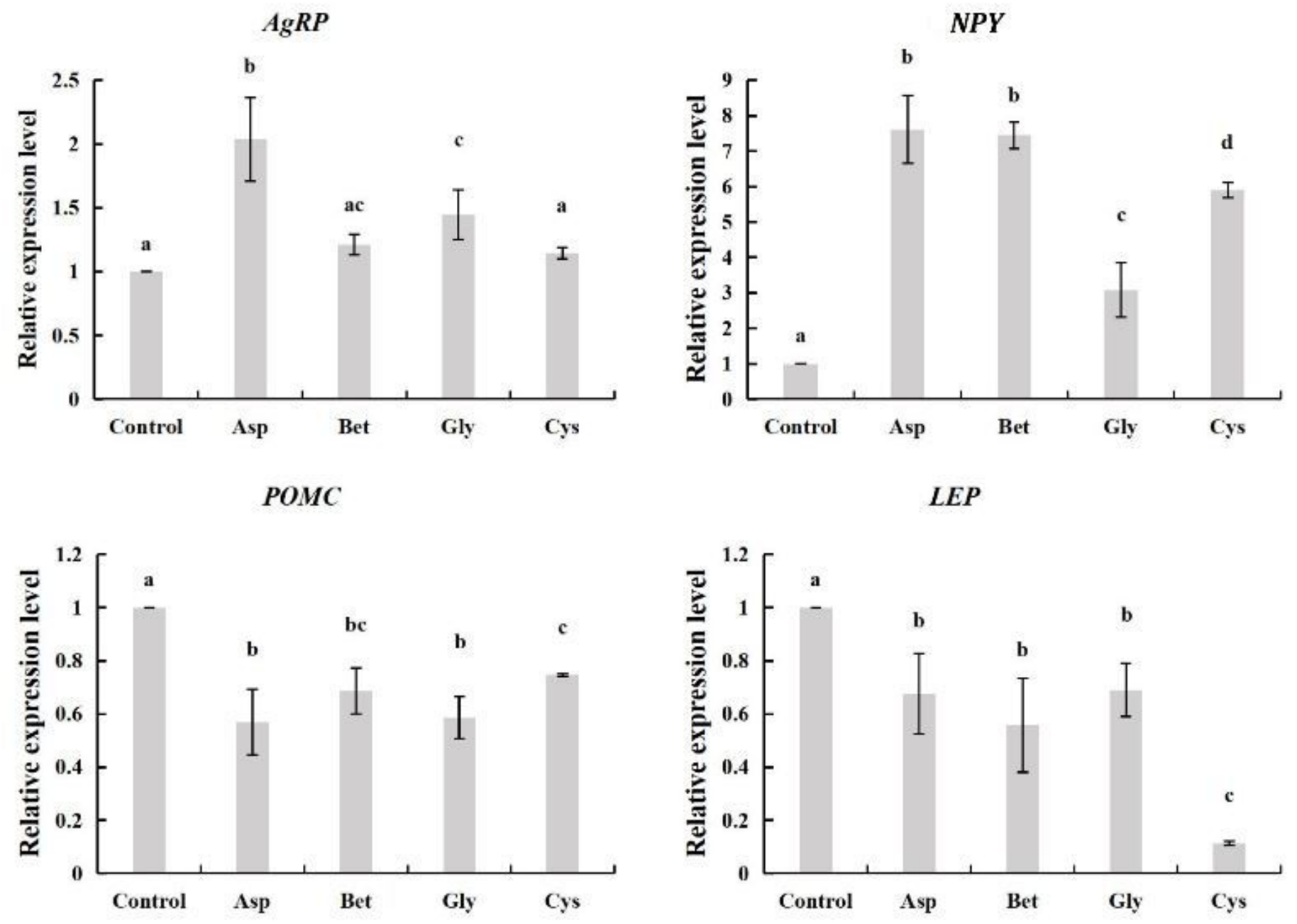

GHRL

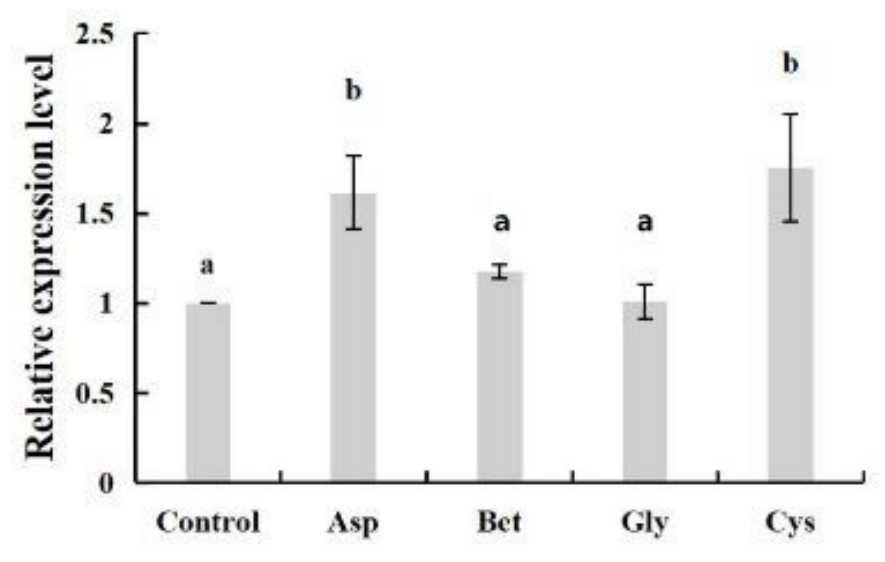

Figure 4

Expression of appetite-related genes in the brain and gut/stomach of silver pomfret exposed to the amino acids aspartic acid (Asp), glycine (Gly), and cysteine (Cys) and positive control betaine (Bet). NPY, AgRP, and POMC expression was detected in the brain; GHRL and LEP expression was detected in the gut/stomach; the results were calculated according to the 2reference gene; Values are means \pm standard deviations $(n=3) ; P<0.05$. 


\section{Supplementary Files}

This is a list of supplementary files associated with this preprint. Click to download.

- Fig.S4.tif

- TableS4.doc

- SuppFig.docx

- Fig.S3.tif

- TableS3.doc

- Fig.S2.tif

- Fig.S1.tif

- Tables2.doc

- Tables1.doc 